\title{
Non-commutative Gross-Neveu model at large $N$
}

\author{
Emil T. Akhmedov, Philip DeBoer and Gordon W. Semenoff \\ Department of Physics and Astronomy, \\ University of British Columbia, \\ Vancouver, British Columbia, Canada V6T 1 Z1.
}

November 5, 2018

\begin{abstract}
The non-commutative $O(N)$ Gross-Neveu model is solved in the large $N$ limit in two and three space-time dimensions. The commutative version of the two dimensional model is a renormalizable quantum field theory, both in a coupling constant expansion and an expansion in $1 / N$. The non-commutative version has a renormalizable coupling constant expansion where ultraviolet divergences can be removed by adjusting counterterms to each order. On the other hand, in a previous work [1] we showed that the non-commutative theory is not renormalizable in the large $N$ expansion. This is argued to be due to a combined effect of asymptotic freedom and the ultraviolet/infrared mixing that occurs in a noncommutative field theory. In the present paper we will elaborate on this result and extend it to study the large $N$ limit of the three dimensional Gross-Neveu model. We shall see that the large $N$ limit of the three dimensional theory is also trivial when the ultraviolet cutoff is removed.
\end{abstract}

\footnotetext{
${ }^{*}$ Permanent Address: Institute of Theoretical and Experimental Physics, B. Cheremushkinskaya 25, 117259 Moscow, Russia
} 


\section{Introduction and Summary}

In a previous paper [1] we discussed the issue of renormalizability of the noncommutative $O(N)$-symmetric Gross-Neveu model in the leading order of a large $N$ expansion. The model is a massless two dimensional field theory of fermions with a four-fermion interaction. It has a dimensionless coupling constant and the commutative version is renormalizable. The coupling constant has an asymptotically free renormalization group flow. As a result, the interaction is large at small momenta. The strong coupling dynamics in the infrared regime leads to spontaneous breaking of chiral symmetry by dynamical generation of a fermion mass [2].

The non-commutative version of this model also has a formally renormalizable perturbation expansion. Non-commutativity improves the ultraviolet limit of the theory slightly in that, order by order in an expansion in the coupling constant, the Feynman diagrams which have ultraviolet divergences are a subset of those which diverged in the commutative theory. It is at least plausible that the ultraviolet divergences can be removed by adjusting counterterms for vertices which have the same form as the vertices in the tree level action [19] [20]. The quantities whose dependence on the ultraviolet cutoff is milder in the noncommutative theory than they were in the commutative theory are more singular at small momenta. This is called ultraviolet/infrared (UV/IR) mixing. It raises the possibility already noted in [15, 16] that, even if the theory is renormalizable in the conventional sense, infrared divergences could upset its consistency.

In our article [1], we addressed this question by examining the large $N$ expansion of the non-commutative theory. This expansion sums a class of Feynman diagrams to all orders. The dimensionless expansion parameter is $1 / N$. We found that, in the large $N$ expansion, because of the singular infrared structure of certain diagrams, the ultraviolet cutoff could not be removed from the effective interactions. Forcibly taking the ultraviolet cutoff to infinity made the effective four-fermion interaction zero. In that limit, the non-commutative $O(N)$ Gross-Neveu model is a trivial, noninteracting field theory.

Our results could be criticized on two grounds. First, though it does not seem relevant to the issue of ultraviolet singularities, being in two dimensions, the model is necessarily space-time non-commutative. When time is not commutative, the action of the non-commutative field theory contains infinite numbers of time derivatives. This makes a Hamiltonian interpretation of the theory difficult and it would be expected to lead to difficulties with unitarity. Though this has little to do with ultraviolet divergences, it could be argued that this will not be a sensible theory in any case because of the unitarity problem.

Secondly, the two dimensional Gross-Neveu model necessarily has spontaneous symmetry breaking and dynamical mass generation. It seems likely that the condensate which breaks the symmetry is a constant, carrying zero momentum. This condensate couples to the zero momentum limit of correlation functions. Because of UV/IR mixing, the correlation functions are singular at zero momentum and the ultraviolet cutoff re-appears as infrared divergences and 
cannot be subtracted by counterterms. If the condensate were not a constant it would not be necessary to use the zero external momentum limit of correlation functions to make the effective action. In that case, the problem with divergences at low momenta could be circumvented. This possibility is difficult to analyze since, thus far, no candidates for a non-constant condensate have been found.

Both of these criticisms can be at least partially addressed by considering the large $N$ limit of the non-commutative three dimensional Gross-Neveu model. In three dimensions, the commutative model has a dimensional coupling constant and is not renormalizable in a conventional perturbation theory which expands order-by order in the coupling. However, it is renormalizable in the $1 / N$ expansion [3, [, 6, 6, ]]. One can examine whether the non-commutative theory is also renormalizable in the same expansion.

Being three dimensional, it is possible to have space-space non-commutativity only, leaving time commutative. There are some general arguments that this should be a unitary theory [8].

Moreover, the commutative three dimensional theory has a second order phase transition between a symmetry breaking ordered phase with dynamically generated fermion mass and a massless, symmetric phase. If the non-commutative theory also has a symmetric phase, it can be studied in a context where generation of a constant condensate is not an issue. A question which we shall address in this paper is whether the large $N$ expansion of the non-commutative theory can be renormalizable in that phase.

The second order phase transition in the commutative three dimensional theory occurs at an infrared stable fixed point of the renormalization group flow of the four-fermion coupling. Existence of the fixed point is a result of the large anomalous dimension of the four-Fermi operator. At the tree level it is an irrelevant dimension four operator. In the large $N$ expansion it behaves like a marginally relevant dimension three operator which flows to a fixed point where the coupling is of order 1 . It is interesting to examine the difference between the behavior of the non-commutative version of this model and the two dimensional model where the dynamical mass generation is the result of the absence of an infrared fixed point.

The great deal of attention that non-commutative field theories have received recently is motivated by the fact that they arise as the low energy limits of string theories with background antisymmetric tensor fields [9, 10, 11, 12, 13, 14]. They retain some of the interesting features of string theory, such as non-locality, which can then be studied in the simpler context of the non-commutative field theory. Since the string theories are consistent quantum mechanical theories, the non-commutative field theories which are their zero slope limits should also be internally consistent. In fact, for some theories, unitarity has been demonstrated explicitly at one-loop order [8]. The issue of renormalizability of these theories asks whether the limit where the string distance scale $\sqrt{\alpha^{\prime}}$ is taken as arbitrarily small produces a field theory with non-trivial interactions.

There are many features of non-commutative field theories which distinguish them from their commutative analogs. One already occurs in scalar field the- 
ory. The non-commutativity, which was at one time suggested as an ultraviolet regularization of quantum field theory, affects the spectrum and interactions at low energy scales, below the momentum scale $1 / \sqrt{|\theta|}$ set by the dimensional parameter $\theta^{\mu \nu}$ and often below the mass scales of the lightest particles already in the model. This has been associated with the phenomenon of UV/IR duality familiar from the behavior of D-branes in string theory [15, 16].

The perturbation expansion of the non-commutative theory is similar to the commutative one [17]. The main difference is that the vertices of the noncommutative theory differ from those in the commutative theory by momentumdependent phases. These phases generally improve the convergence of loop integrals.

In non-commutative field theory, there is a fat-graph representation of the perturbation theory in which the Feynman diagrams can be classified according to the genus of the Riemann surface on which they could be drawn without crossing any lines. Diagrams can be classified according to whether they are planar or non-planar graphs [17]. The Feynman integrands of planar graphs are as they were in the commutative case. The integrands of non-planar graphs are modified by phases containing external and internal loop momenta. The presence of these phases improves the high-momentum behavior of Feynman integrals. The most dramatic effect occurs in diagrams which are ultraviolet divergent. Planar diagrams diverge and must be defined using a high momentum cutoff, $\Lambda$, as was the case in the commutative theory. In the non-planar diagrams the loop integrations are affected by phases and generally converge, the ultraviolet cutoff being replaced by an effective cutoff $\Lambda_{\text {eff }}(p)=1 / \sqrt{1 / \Lambda^{2}+(\theta p)^{2} / 4}$. For any momentum in the range $p>1 /(\theta \Lambda)$, this effective cutoff has a finite limit, $\Lambda_{\text {eff }} \sim 1 / 2|\theta p|$, as $\Lambda \rightarrow \infty$.

For example, at one loop order in 4 -dimensional $\phi^{4}$-theory, the radiative correction to the scalar self-energy in the commutative version is a quadratically divergent constant coming from a tadpole diagram. In the non-commutative theory, there are two contributions, coming from a planar and a non-planar tadpole. The planar graph is again a quadratically divergent constant, as it was in the commutative case. The non-planar graph, even thought it is a tadpole, turns out to be a function of external momentum. At small momentum, 15, 16]

$$
\Gamma^{(2)}(p)=\frac{g^{2}}{48 \pi^{2}}\left(\Lambda^{2}-m^{2} \ln \frac{\Lambda^{2}}{m^{2}}\right)+\frac{g^{2}}{96 \pi^{2}}\left(\Lambda_{\mathrm{eff}}^{2}(p)-m^{2} \ln \frac{\Lambda_{\mathrm{eff}}^{2}(p)}{m^{2}}\right)+\ldots
$$

Here, $m$ is the scalar field mass and $g^{2}$ is the dimensionless $\phi^{4}$ coupling constant. The second term has a pole at very low momenta. It also has a logarithmic cut singularity at small momenta. These have been argued to arise from new degrees of freedom with exotic dispersion relations or perhaps propagating in higher dimensions [16]. They have also been argued to lead to exotic translation non-invariant "striped" phases of scalar field theory [18].

Another place where ultraviolet divergences occur is in the renormalization of dimensionless coupling constants. The leading corrections to the coupling 
constant in 4-dimensional $\phi^{4}$-theory are logarithmically divergent. The small momentum limit of the 4-point function was computed in 15] as

$$
\begin{aligned}
\Gamma^{(4)}(p, q, r, s)=g^{2}- & \frac{g^{2}}{2 \cdot 2^{5} \pi^{2}}\left\{2 \ln \frac{\Lambda^{2}}{m^{2}}+\ln \frac{1}{m^{2}(\theta p)^{2}}+\ln \frac{1}{m^{2}(\theta q)^{2}}+\right. \\
+ & \ln \frac{1}{m^{2}(\theta r)^{2}}+\ln \frac{1}{m^{2}(\theta s)^{2}}+\ln \frac{1}{m^{2}(\theta(q+r))^{2}}+ \\
& \left.+\ln \frac{1}{m^{2}(\theta(q+s))^{2}}+\ln \frac{1}{m^{2}(\theta(r+s))^{2}}\right\}+\ldots
\end{aligned}
$$

$(p+q+r+s=0)$ The first contribution is from planar diagrams and is similar to that in the commutative theory, with slightly different coefficient. The other contributions are from non-planar diagrams and they depend explicitly on the parameter $\theta$.

What is remarkable about (2) is that, in spite of the mass gap of the bare scalar field, the effective coupling constant is logarithmically singular at small momentum. This logarithmic singularity is similar to the one which occurs in the same function at large momentum (with $m^{2}(\theta p)^{2}$ replaced by $p^{2} / \Lambda^{2}$ ). It becomes large and ruins the perturbation expansion in both the regimes of very large and very small momentum transfers.

This behavior is radically different from that of commutative field theory where the running of coupling constants at low momenta is cutoff by mass scales and is frozen at the mass scale of the lightest matter particles which participate in the interaction. For example, in quantum electrodynamics which, like $\phi^{4}$ theory is infrared free, the coupling constant runs at energies much larger than the electron mass, but as the energy is lowered, it freezes at the value $e^{2} / 4 \pi \sim$ $1 / 137$ and is the same at all lower energy scales. In a non-commutative theory, it appears that masses do not cutoff the running of coupling constants. We emphasize that this is a non-perturbative issue, which occurs in addition to the perturbative renormalizability of non-commutative field theories which has recently been examined in detail [19, 20, 21].

This unusual running of the coupling at low momenta is a mirror of the running of the coupling at high momenta which necessarily occurs when the momentum scale is larger than any dimensional couplings or masses but still less than the UV cutoff. The UV/IR mixing in non-commutative field theory seems to mirror that running with a similar low energy behavior, regardless of the existence of masses for the particles.

It is interesting to compare the Gross-Neveu model with the four-dimensional $O(N)$ vector model with action

$$
S=\int d^{4} x\left\{\frac{1}{2} \partial \phi^{i} \cdot \partial \phi^{i}+\frac{\lambda}{8 N}\left(\phi^{i} \phi^{i}\right)^{2}\right\}
$$

In this model the infrared rather than asymptotically free running of the effective coupling constant can lead to a ground state which breaks translation invariance, but is otherwise apparently consistent interacting theory when the cutoff is large. 
The large $N$ expansion is solved by introducing an auxiliary field $\sigma$,

$$
S=\int d^{4} x\left\{\frac{1}{2} \partial \phi^{i} \cdot \partial \phi^{i}+\frac{i}{2} \sigma \phi^{i} \phi^{i}+\frac{N}{2 \lambda} \sigma^{2}\right\}
$$

Then, in the leading order in large $N$, the small momentum limit of the quadratic term in the effective action for $\sigma$

$$
\frac{N}{2} \int d^{4} p \sigma^{*}(p)\left(\frac{1}{\lambda}+c \ln \frac{\Lambda^{2}}{\mu^{2}}+c^{\prime} \ln \frac{\Lambda_{\text {eff }}^{2}(p)}{\mu^{2}}+\ldots\right) \sigma(p)
$$

where $\sigma(p)$ is the Fourier transform of $\sigma(x)$. Here $c$ and $c^{\prime}$ are positive constants. They are positive because the beta function of 4-dimensional scalar field theory is positive. It is infrared free. The first logarithm on the right-hand-side comes from a planar diagram, the second from a non-planar diagram. Only the small momentum limit is presented. The contribution of the planar diagram would go to zero at large momentum. The inverse of the bare $\phi^{4}$-coupling constant $\lambda$ must be adjusted to cancel the UV singularity. If we tune $\lambda$ to absorb the singularity and put the cutoff to infinity, we obtain an expression like

$$
\frac{N}{2} \int d^{4} p \sigma^{*}(p)\left(\frac{1}{\tilde{\lambda}}+c^{\prime} \ln \frac{1}{2|\theta p| \mu^{2}}+\ldots\right) \sigma(p)
$$

To find a phase transition, we lower $1 / \tilde{\lambda}$ to negative values. Eventually when $1 / \tilde{\lambda}$ is negative enough, $\sigma$ will have a tachyonic mode. This should first occur for modes at some finite momentum and there will be a condensate of $\sigma(p)$ where $p$ is non-zero and $\langle\sigma(x)\rangle$ is not a constant. This is a different mechanism for breaking of translation symmetry from the one which was found in 18.

Now, what happens in the Gross-Neveu model? Let us focus on the two dimensional case. There, the auxiliary scalar field has the quadratic term in its effective action

$$
\frac{N}{2} \int d^{2} p \sigma^{*}(p)\left(\frac{1}{\lambda}-g \ln \frac{\Lambda^{2}}{\mu^{2}}-g^{\prime} \ln \frac{\Lambda_{\text {eff }}^{2}(p)}{\mu^{2}}+\ldots\right) \sigma(p)
$$

where $g$ and $g^{\prime}$ are positive. Note that the coefficients of the logarithms are negative. This is a result of the fact that the beta function is negative and the theory is asymptotically free. Again, we can add a counterterm to cancel the UV singularity of the first logarithm, to get

$$
\frac{N}{2} \int d^{2} p \sigma^{*}(p)\left(\frac{1}{\tilde{\lambda}}-g^{\prime} \ln \frac{\Lambda_{\text {eff }}(p)}{\mu^{2}}\right) \sigma(p)
$$

Now, the infinite $\Lambda$ limit gives a finite expression, but there is a dramatic difference from the infrared free case, the term is always going to be negative for small enough momenta and always has a minimum at zero momentum. Then, at the minimum, the quadratic term in the effective action for $\sigma$ diverges. If we add a counterterm to make it finite at zero momentum, then it would have a divergence at finite momentum. This is what leads to triviality. The three dimensional case has similar behavior, even in the phase where there should be no condensate. The details are in the remainder of this paper. 


\subsection{Notation}

On either non-commutative Euclidean space, $R^{d}$, or Minkowski space, $M^{d}$, the coordinates have the algebra

$$
\left[x^{\mu}, x^{\nu}\right]=-i \theta^{\mu \nu}
$$

where $\theta^{\mu \nu}$ is an antisymmetric matrix. Being anti-symmetric, it must have even rank.

Equivalent to imposing the algebra (3) on the coordinates, non-commutativity can also be implemented by replacing the usual product for multiplying functions by the associative, non-commutative and non-local $*$-product,

$$
f(x) * g(x) \equiv \lim _{y \rightarrow x} \exp \left(-\frac{i}{2} \theta^{\mu \nu} \frac{\partial}{\partial x^{\mu}} \frac{\partial}{\partial y^{\nu}}\right) f(x) g(y)
$$

Under an integral, we can always use integration by parts to remove one of the $*$-products and replace it with an ordinary product,

$$
\int f_{1} * f_{2} * \ldots * f_{n-1} * f_{n}=\int f_{1}\left(f_{2} * \ldots * f_{n-1} * f_{n}\right)
$$

For this reason, the multiplication of functions under an integral has cyclic symmetry

$$
\int f_{1} * f_{2} * \ldots * f_{n-1} * f_{n}=\int f_{n} * f_{1} * f_{2} * \ldots * f_{n-1}
$$

Note that in two dimensions non-commutativity does not break Lorenz invariance, $\theta_{\mu \nu}=\theta \cdot \epsilon_{\mu \nu}$. Also, in 1+1-dimensional Minkowski space, it would not be possible to set $\theta^{0 i}$ to zero, unless one set the entire matrix to zero so time is necessarily non-commutative if the space is.

In three dimensions we can always take the space coordinates as non-commuting and leave the time commutative by choosing $\theta^{0 i}=0$. In this paper we will assume that this is the case.

\section{The non-commutative $\mathrm{O}(\mathrm{N})$ Gross-Neveu model}

In this section we will fix the notation and define the models which we will examine in some detail in the following Sections. We review some of the techniques for solving a vector model in the large $N$ limit. The Euclidean action of the non-commutative Gross-Neveu model is

$$
S[\psi]=-\int d^{D} x\left\{\frac{1}{2} \sum_{j=1}^{N} \bar{\psi}^{j} \gamma \cdot \partial \psi^{j}+\frac{\lambda}{8 N} \sum_{i j=1}^{N} \bar{\psi}^{i} * \psi^{i} * \bar{\psi}^{j} * \psi^{j}\right\}
$$

where $D$ is the dimension of space-time, which we assume to have Euclidean signature. 
The spinors $\psi^{i}$ are Majorana fermions. They obey the constraint $\psi=C \psi^{*}$ where $C$ is the charge conjugation matrix. We use Majorana, rather than Dirac fermions in order to generate non-planar diagrams at the leading order in large $N$. In two dimensions, the Majorana spinors have two components and there is a choice of Dirac matrices for which the spinor is real. In three dimensions, we could take them as having either two or four components and, for concreteness, we will take them to have two components. The distinction between these two choices is not important for the arguments of this paper.

In three dimensions, with two-component spinors, the condensate that we discuss will break space-time parity.

In two dimensions, the kinetic term in (7) has $O(N)_{L} \times O(N)_{R}$ chiral symmetry. The interaction term breaks this to $O(N) \times Z_{2}$ where the $O(N)$ is a diagonal subgroup of $O(N)_{L} \times O(N)_{R}$ and the $Z_{2}$ is a discrete chiral symmetry. Under this residual chiral transformation, $\psi \rightarrow \gamma^{5} \psi$ with $\gamma^{5}=i \gamma^{1} \gamma^{2}$. This symmetry forbids fermion mass and, for the fermions to become massive, the $Z_{2}$ symmetry must be spontaneously broken. The condensate $\langle\bar{\psi} \psi\rangle$ is an order parameter for this symmetry breaking.

In three dimensions, with 2-component spinors, the kinetic and interaction term have $O(N)$ symmetry. The condensate $\langle\bar{\psi} \psi\rangle$ and accompanying fermion mass generation that we are interested in studying breaks parity.

All products in $(\overline{7})$ are $*$ products, as defined in (伍). When we set total derivative terms in the action (7) to zero, in each term, because we can integrate by parts, one of the *-products is always equal to an ordinary product. For this reason only the ordinary product occurs in quadratic terms. The quartic term can be written as $\int(\bar{\psi} * \psi)^{2}$. We shall use this fact when we introduce an auxiliary field.

In the non-commutative theory, there is another interaction vertex of the form

$$
\int d x \sum_{i, j=1}^{N} \bar{\psi}^{i} * \psi^{j} * \bar{\psi}^{i} * \psi^{j}
$$

which one could add to the action density. Because of the non-commutativity of the *-product it is not equivalent to the one that is already in (7). We have chosen not to add this vertex because it is technically more complicated to deal with than the one in (7). This vertex should not alter solvability of the model in the large $N$ limit. However, it seems that there is no local master field when it is present and it is likely that the ground state has broken translation invariance. We shall not examine this issue further in this paper. Here, we note that, at least in the leading order in large $N$, we shall find that the model (7) seems to be consistent without this additional vertex - as a vertex of that form does not seem to be generated dynamically. 


\subsection{The effective action}

It is useful consider the generating functional for correlation functions of the spinor field,

$$
Z[\eta]=\int[d \psi] \exp \left(-S[\psi]+\int d^{D} x \bar{\eta}(x) \psi(x)\right)
$$

where $\eta(x)$ is an anti-commuting classical Majorana spinor. The correlation functions are obtained by taking functional derivatives of this generating functional,

$$
\left\langle\psi\left(x_{1}\right) \ldots \psi\left(x_{n}\right)\right\rangle=\left.\frac{1}{Z[0]} \frac{\delta}{\delta \bar{\eta}\left(x_{1}\right)} \ldots \frac{\delta}{\delta \bar{\eta}\left(x_{n}\right)} Z[\eta]\right|_{\eta=0}
$$

As usual, connected correlation functions are generated by the logarithm of the partition function,

$$
W[\eta]=-\ln (Z[\eta])
$$

From this, it is convenient to convert to a generating functional for one-fermion irreducible correlation functions. This is done using a Legendre transform. We define the 1-point function in the presence of the source by

$$
\psi_{0}(x) \equiv<\psi(x)>=-\frac{\delta}{\delta \bar{\eta}(x)} W[\eta]
$$

Then, we consider the Legendre transform

$$
\Gamma\left[\psi_{0}\right]=W[\eta]+\int d^{D} x \bar{\eta}(x) \psi_{0}(x)
$$

From this equation, we see that

$$
\frac{\delta}{\delta \psi_{0}(x)} \Gamma\left[\psi_{0}\right]=-\bar{\eta}(x)
$$

is an equation which determines the classical expectation value of $\psi$ induced by the source. $\Gamma\left[\psi_{0}\right]$ is sometimes called the effective action. Higher functional derivatives of $\Gamma\left[\psi_{0}\right]$ by $\psi_{0}$ give one-fermion irreducible correlation functions. The connected correlation functions can be reconstructed from these by combinatorics and, in turn, one can reconstruct the full correllators.

\section{$2.2 \quad$ Large $\mathrm{N}$}

In order to solve the large $N$ limit of the model (7), it is convenient to introduce an auxiliary field so that the action is (where now we include the source term in the action)

$$
S[\psi, \phi, \eta]=\int d^{D} x\left\{-\frac{1}{2} \sum_{j=1}^{N} \bar{\psi}^{j}(\gamma \cdot \partial+* \phi *) \psi^{j}+\frac{N}{2 \lambda} \phi^{2}-\bar{\eta} \psi\right\}
$$


The original action (7) is recovered by doing the Gaussian integral of $\phi$ in the partition function,

$$
Z[\eta]=\int[d \psi d \phi] \exp (-S[\psi, \phi, \eta])
$$

Alternatively, this is now a Gaussian integral for $\psi$ and we can integrate out $\psi$ to get the non-local scalar field theory with action

$$
S[\phi, \eta]=-\frac{N}{2} \operatorname{Tr} \ln (\gamma \cdot \partial+* \phi *)+\int\left(\frac{N}{2 \lambda} \phi^{2}-\frac{1}{2} \bar{\eta} \frac{1}{\gamma \cdot \partial+* \phi *} \eta\right)
$$

where $* \phi *$ denotes multiplication using the $*$-product. Some care will be required in defining the fermion determinant with $* \phi *$ present. We will discuss the details below.

In the large $N$ limit, the remaining functional integral can be evaluated by saddle point approximation. For this, we must find a minimum of (16) as a functional of $\phi$. Then, the large $N$ limit of the generating functional for connected correllators is given by

$$
W[\eta]=\inf _{\phi}:-\frac{N}{2} \operatorname{Tr} \ln (\gamma \cdot \partial+* \phi *)+\int\left(\frac{N}{2 \lambda} \phi^{2}-\frac{1}{2} \bar{\eta} \frac{1}{\gamma \cdot \partial+* \phi *} \eta\right)
$$

where the infimum is taken while holding $\eta$ fixed.

This can be converted to the generating functional for one-fermion-irreducible graphs by taking the explicit Legendre transform. The result is the elegant expression

$$
\Gamma\left[\psi_{0}\right]=\inf _{\phi}:-\frac{N}{2} \operatorname{Tr} \ln (\gamma \cdot \partial+* \phi *)+\int\left(\frac{N}{2 \lambda} \phi^{2}-\frac{1}{2} \bar{\psi}_{0} \gamma \cdot \partial \psi_{0}+\frac{1}{2} \phi \bar{\psi}_{0} * \psi_{0}\right)
$$

where now the infimum over $\phi$ must be found while holding $\psi_{0}$ fixed. Then, when we find the infimum, it will depend on $\psi_{0}$. Substituting it back into (18) gives a functional of $\psi_{0}$ whose functional derivatives by $\psi_{0}$ are the one-fermion irreducible correlation functions to leading order in the large $N$ expansion. The leading contribution to the correlator with $\mathrm{n}$ external Fermion legs is order $N^{1-n}$ and is readily obtained from this expression. It is possible to obtain higher orders in $1 / N$ by finding corrections to the saddle point approximation that we have used here. This is a systematic procedure.

It is clear that $\eta$ and $\psi_{0}$ are related in a complicated way. We will always assume that $\psi_{0}$ goes to zero when $\eta$ goes to zero. Since $\Gamma\left[\psi_{0}\right]$ is an even function of $\psi_{0}$, when $\eta=0, \psi_{0}=0$ is always a solution of (13).

We will restrict our search for infima in (18) to those which give translation invariant ground states, i.e. to where the function $\phi$ which minimizes (18) goes to a constant when $\psi_{0}$ goes zero. At this point, we do not know whether there are translation non-invariant solutions which have smaller action than the translation invariant solution that we find. We will not address this issue in this Paper. Since we will find no tachyons in the spectrum, the solution that we consider is at least 
a local minimum if not an infimum. If $\phi=M$ is a constant, its $*$-product with other functions reduces to an ordinary product. Then, setting $\psi_{0}$ to zero for the moment, we can readily evaluate (18) with $\phi=M$. The function which must be minimized to find the vacuum value of $M$ is the effective potential

$$
V_{\text {eff }}(M)=-\frac{N}{2} \frac{1}{\mathrm{v}} \operatorname{Tr} \ln (\gamma \cdot \partial+M)+\frac{N}{2 \lambda} M^{2}
$$

where we have divided by the space-time volume, $\mathrm{v}$. The trace is evaluated as

$$
V_{\text {eff }}=-\frac{N d}{4} \int \frac{d^{D} p}{(2 \pi)^{D}} \ln \left(p^{2}+M^{2}\right)+\frac{N}{2 \lambda} M^{2}
$$

where $d$ is the dimension of the Gamma matrices. An ultraviolet cutoff is needed to evaluate this integral. After a cutoff is introduced, it can easily be evaluated in 2 and 3 dimensions. The value of $M$ which minimizes this integral is the vacuum expectation value of $\phi$ when the sources are set to zero. The gap equation either has the solution $M=0$ or $M$ solves the equation

$$
\frac{1}{\lambda}=\frac{d}{2} \int \frac{d^{D} p}{(2 \pi)^{D}} \frac{1}{p^{2}+M^{2}}
$$

Then we must check whether the solution of (21) or $M=0$ is the stable solution.

\subsection{Non-commutative fermion determinant}

When $\phi$ is not a constant, we must take some care in evaluating the fermion determinant in order to correctly take into account the $*$-products. If $\phi$ has the form $\phi=M+\delta \phi$, where $M$ is a constant, the determinant is defined by the expression

$$
-\frac{1}{2} \ln \operatorname{Tr}(\gamma \cdot \partial+* \phi *) \equiv \sum_{n=1}^{\infty} \frac{1}{n !} \int \delta \phi\left(x_{1}\right) \ldots \delta \phi\left(x_{n}\right) \tau\left(x_{1}, \ldots, x_{n}\right)
$$

where

$$
\tau\left(x_{1}, \ldots, x_{n}\right)=-\left(\frac{1}{2}\right)^{n}\left\langle\bar{\psi}\left(x_{1}\right) * \psi\left(x_{1}\right) \ldots \bar{\psi}\left(x_{n}\right) * \psi\left(x_{n}\right)\right\rangle_{0}^{\mathrm{conn} .}
$$

The expectation values on the right-hand-side of this equation are connected correlators of free fermions with mass $M$. The correlators of free fermions of mass $M$ are defined by,

$$
<\psi\left(x_{1}\right) \ldots \psi\left(x_{n}\right)>_{0}=\frac{\int[d \psi] \psi\left(x_{1}\right) \ldots \psi\left(x_{n}\right) e^{\int \frac{1}{2} \bar{\psi}(\gamma \cdot \partial+M) \psi}}{\int[d \psi] e^{\int \frac{1}{2} \bar{\psi}(\gamma \cdot \partial+M) \psi}}
$$

From these, one should choose the connected ones to form the correlators on the right-hand-side of (23). 
Then, the equation which determines $\delta \phi(x)$ is

$$
\sum_{1}^{\infty} \frac{1}{n !} \int d x_{1} \ldots d x_{n} \tau\left(x, x_{1}, \ldots, x_{n}\right) \delta \phi\left(x_{1}\right) \ldots \delta \phi\left(x_{n}\right)+\frac{1}{\lambda} \delta \phi(x)+\frac{1}{2 N} \bar{\psi}_{0}(x) * \psi_{0}(x)=0
$$

The solution of this equation should then be substituted back into (18) to find the generating functional for irreducible correlators.

\subsection{Effective four-fermion coupling}

The equation (25) which determines $\delta \phi(x)$ can be solved iteratively.

To get the leading order, we define the Fourier transform

$$
\tau\left(x_{1}, x_{2}\right)=\int \frac{d^{2} q}{(2 \pi)^{2}} \tau(q) e^{i q \cdot\left(x_{1}-x_{2}\right)}
$$

We shall also find it a useful short-hand to write $\tau\left(x_{1}, x_{2}\right)$ as a differential operator

$$
\tau\left(x_{1}, x_{2}\right)=\tau(-i \partial) \delta\left(x_{1}-x_{2}\right)
$$

Then,

$$
\delta \phi(x)=-\frac{\lambda}{1+\lambda \tau(-i \partial)} \frac{1}{2 N} \bar{\psi}_{0}(x) \psi_{0}(x)+\ldots
$$

where corrections are terms of second and higher order in $\bar{\psi} \psi$.q Substituting this back into the effective action obtains

$$
\Gamma\left[\psi_{0}\right]=-\int\left(\frac{1}{2} \bar{\psi}_{0}(\gamma \cdot \partial+M) \psi_{0}+\frac{1}{8 N} \bar{\psi}_{0} * \psi_{0} \frac{\lambda}{1+\lambda \tau(-i \partial)} \bar{\psi}_{0} * \psi_{0}+\ldots\right)
$$

The leading terms in this effective action have the same form as the original action with the coupling constant replaced by an effective coupling which depends on the momentum transfer $p$ and is given by

$$
\lambda^{\mathrm{eff}}(p)=\frac{\lambda}{1+\lambda \tau(p)}
$$

Thus, to find the effective four-fermion interaction, we must find the twopoint correlator $\tau\left(x_{1}, x_{2}\right)$. Out of all the terms in (25), it is only this, leading one which is ultraviolet divergent in either two or three spacetime dimensions. It is therefore the only one that will be affected by UV/IR mixing in this leading order of the large $N$ expansion.

\footnotetext{
${ }^{1}$ Here we are distinguishing the function $\tau$ from its Fourier transform by writing its space version with two arguments and its Fourier transform in momentum space with one argument. Which of the two we mean in a particular place should always be clear from the context.
} 


\subsection{Summary}

Let us review the procedure to be followed to get the effective coupling constant. We must first solve the gap equation (21) for the fermion mass $M$ and decide whether this solution, if it exists, or the trivial solution $M=0$ is stable. The stable solution is a global minimum of the effective potential in (20). Then we must choose the stable solution for $M$ and use it to compute the function $\tau(p)$ using equation (23). Using those two results, we find the effective coupling constant using equation (26).

\section{Results in two dimensions}

In two dimensions, the effective potential is

$$
V_{\text {eff }}=-\frac{N}{8 \pi}\left(M^{2} \ln \frac{\Lambda^{2}}{M^{2}}+M^{2}\right)+\frac{N}{2 \lambda} M^{2}
$$

This potential applies to either the commutative or the non-commutative theory. It must be minimized in order to find the physical value of $M$. In two dimensions, it always has a doubly degenerate global minimum for non-zero $M$ which occurs when the gap equation is solved,

$$
\frac{1}{\lambda}=\frac{1}{4 \pi} \ln \frac{\Lambda^{2}}{M^{2}}
$$

Both $M$ and $-M$ are solutions of this equation, reflecting the chiral symmetry.

The equation (28) is a statement of dimensional transmutation: when (28) is substituted for the coupling constant in a physical expression in a renormalizable theory, the dependence of the cutoff $\Lambda$ cancels and the parameter remaining is the mass scale, $M$, in this case the dynamically generated fermion mass. Thus, the bare dimensionless coupling $\lambda$ and ultraviolet cutoff $\Lambda$ are traded for a dimensional parameter, $M$. The theory doesn't have a coupling constant, instead it has a mass scale and is weakly coupled for processes with momenta which are greater than $M$ and strongly coupled when the momentum is less than $M$.

We will see this explicitly in the effective coupling constant for the commutative theory, there $\Lambda$ and $\lambda$ disappear from the effective coupling constant once the gap equation is solved. They are replaced by the mass scale, $M$.

Although we will not use it in the following, we could introduce a renormalized coupling constant so that, substitution into (27) and (28) we would remove the ultraviolet divergences,

$$
\frac{1}{\lambda}=\frac{1}{\lambda_{\operatorname{ren}}(\mu)}+\frac{1}{4 \pi} \ln \frac{\Lambda^{2}}{\mu^{2}}
$$

Then, notice that, if we hold $\lambda_{\text {ren }}(\mu)$ fixed as we put the cutoff $\Lambda$ to infinity, the bare coupling constant $\lambda$ goes to zero. This is another manifestation of asymptotic freedom. On the other hand, if we hold the bare coupling $\lambda$ and the cutoff $\Lambda$ fixed as we take the renormalization scale $\mu$ small, the renormalized 
coupling $\lambda_{\text {ren }}(\mu)$ increases and goes to infinity at some small scale. This is infrared slavery and the infrared Landau pole. Of course, the true running of the effective coupling constant is cutoff by mass generation.

\subsection{Commutative model}

To see how this works, we first consider the commutative theory. We can do this by setting the matrix $\theta^{\mu \nu}$ to zero, which amounts to treating all of the $*$-products in the above formulae as ordinary products. In this case,

$$
\tau_{\mathrm{c}}(p)=-\frac{1}{2 \pi}\left(\ln \frac{\Lambda e^{1-\gamma}}{M}-\frac{\sqrt{1+\frac{p^{2}}{4 M^{2}}}}{\frac{p}{2 M}} \ln \left(\sqrt{1+\frac{p^{2}}{4 M^{2}}}+\frac{p}{2 M}\right)\right)
$$

where the subscript, c, denotes commutative, $\gamma$ is Euler's constant. Here and below we use the same regularization as in [15] and [16]. Then, in this case, the effective coupling constant is

$$
\lambda_{\mathrm{c}}^{\mathrm{eff}}(p)=\frac{2 \pi}{\frac{\sqrt{1+\frac{p^{2}}{4 M^{2}}}}{\frac{p}{2 M}} \ln \left(\sqrt{1+\frac{p^{2}}{4 M^{2}}}+\frac{p}{2 M}\right)+\gamma-1}
$$

Here, we have used the gap equation (28) to eliminate the coupling constant. Note that it also cancels the ultraviolet cutoff, leaving only the mass parameter, $M$. For large momenta, $p>>M$, the effective coupling

$$
\lambda_{\mathrm{c}}^{\mathrm{eff}}(p) \approx \frac{2 \pi}{\ln (p / M)}
$$

is small, as we expected. As we lower the momentum to small momenta, $p<<M$, it increases and stops increasing when the momentum gets to the scale $M$, where it freezes at

$$
\lambda_{\mathrm{c}}^{\mathrm{eff}}(p) \approx 2 \pi / \gamma
$$

\subsection{Non-commutative model}

Now, let us examine the non-commutative theory. There, $\tau(p)$ gets a contribution from both a planar and a non-planar graph. The planar diagram contributes

$$
\tau_{\mathrm{nc}}^{\mathrm{pl}}(p)=-\frac{1}{4 \pi}\left(\ln \frac{\Lambda e^{1-\gamma}}{M}-\frac{\sqrt{1+\frac{p^{2}}{4 M^{2}}}}{\frac{p}{2 M}} \ln \left(\sqrt{1+\frac{p^{2}}{4 M^{2}}}+\frac{p}{2 M}\right)\right)
$$

This planar diagram is not affected by the non-commutativity. That is why the result is half of the commutative contribution (29).

There is also a non-planar diagram. It depends on the non-commutativity parameter in a non-trivial way,

$$
\tau_{\mathrm{nc}}^{\mathrm{nonpl}}(p)=-\frac{1}{4 \pi} K_{0}\left(2 M \sqrt{\left[\theta^{2} q^{2} / 4+1 / \Lambda^{2}\right]}\right)+
$$




$$
+\frac{1}{4 \pi}\left(M^{2}+\frac{q^{2}}{4}\right) \int_{0}^{1} d \alpha \int_{0}^{\infty} d \rho \exp \left\{-\rho\left(M^{2}+\alpha(1-\alpha) q^{2}\right)-\frac{(\theta q)^{2}}{4 \rho}\right\} .
$$

Here $K_{0}(z)$ is the modified Bessel function. Note that, even though the planar contribution is divergent, for non-zero momenta the non-planar one has a finite limit as the ultraviolet cutoff is put to infinity. The correlator $\tau_{\mathrm{nc}}(p)$ in the non-commutative theory is the sum of the above two contributions

$$
\tau_{\mathrm{nc}}(p)=\tau_{\mathrm{nc}}^{\mathrm{pl}}(p)+\tau_{\mathrm{nc}}^{\mathrm{nonpl}}(p)
$$

The effective four point coupling of the fermions with momentum transfer $p$ is

$$
\lambda^{\mathrm{eff}}(p)=\frac{1}{\frac{1}{\lambda}+\tau_{\mathrm{nc}}^{\mathrm{pl}}(p)+\tau_{\mathrm{nc}}^{\mathrm{nonpl}}(p)}
$$

When we substitute the cut-off-dependent expression (28) for $1 / \lambda$ into (31), the UV cutoff dependence does not cancel. If for any momentum in the range $p>$ $1 / \theta \Lambda$, the effective coupling $\lambda_{\text {eff }}(p)$ goes to zero as $\Lambda$ is taken to infinity.

Let us find UV behavior of (31). In the limit when $p^{2}>>4 M^{2}, p^{2}>>$ $1 /(\theta M)^{2}$ (we always assume that $p^{2}<<\Lambda^{2}$ and $M^{2}<<\Lambda^{2}$ ) we have

$$
\tau_{1}(p) \approx-\frac{1}{8 \pi} \ln \frac{\Lambda^{2}}{p^{2}} \quad \text { and } \quad \tau_{2}(p) \sim e^{-\theta M p}
$$

Thus

$$
\lambda^{\mathrm{eff}}(p) \approx \frac{1}{\frac{1}{\lambda}-\frac{1}{8 \pi} \ln \frac{\Lambda^{2}}{p^{2}}}=\frac{8 \pi}{\ln \frac{\Lambda^{2} p^{2}}{M^{4}}},
$$

where $\lambda$ is eliminated using (28). On the other hand, when $p^{2}<<1 /\left(\theta^{2} M^{2}\right)$, $p^{2}<<4 M^{2}$ and $p^{2}>>1 /(\theta \Lambda)^{2}$ we can approximate the above expressions by

$$
\tau_{\mathrm{nc}}^{\mathrm{pl}}(p) \approx-\frac{1}{8 \pi} \ln \frac{\Lambda^{2}}{M^{2}} \quad \text { and } \quad \tau_{\mathrm{nc}}^{\mathrm{nonpl}}(p) \approx-\frac{1}{8 \pi} \ln \left(\theta^{2} p^{2} M^{2}\right)
$$

Hence

$$
\lambda^{\mathrm{eff}}(p) \approx \frac{1}{\frac{1}{\lambda}-\frac{1}{8 \pi} \ln \frac{\Lambda^{2}}{M^{2}}-\frac{1}{8 \pi} \ln \left(\theta^{2} p^{2} M^{2}\right)}=\frac{8 \pi}{\ln \left(\Lambda^{2} \theta^{2} p^{2}\right)} .
$$

For momenta above $p \sim 1 / \theta \Lambda$ the last expression depends on the cutoff and for finite, nonzero momentum it goes to zero as the cutoff goes to infinity.

Thus, we find that, in the non-commutative theory, renormalization does not remove the cutoff dependence of the effective four-fermion interaction. The interaction is suppressed by an inverse power of the ultraviolet cutoff and goes to zero - rendering the theory trivial - as the cutoff is put to infinity. 


\subsection{What if we choose $1 / \lambda$ so that the cutoff cancels?}

It is the solution of the gap equation (28) which dictated the cutoff dependence of $\lambda$. If we choose a different cutoff dependence for $1 / \lambda$, the gap equation would not be satisfied. This means that the system would be unstable. We would see this immediately in the effective coupling constant (31) - in fact the stability condition for quadratic fluctuations of $\phi(x)$ in the action is the positivity condition for inverse of the effective coupling.

For example, we could remove the cutoff dependence by choosing

$$
\frac{1}{\lambda}=\frac{1}{\tilde{\lambda}(\mu)}+\frac{1}{8 \pi} \ln \frac{\Lambda^{2}}{\mu^{2}}
$$

Then, we can take the cutoff to infinity. We find, at very small momenta

$$
\lambda^{\mathrm{eff}}(p) \approx \frac{1}{\frac{1}{\tilde{\lambda}(\mu)}+\frac{1}{8 \pi} \ln \left(\mu^{2} p^{2} \theta^{2} / 4\right)}
$$

This effective coupling exhibits an infrared Landau pole at a small value of the momentum squared,

$$
p_{L}^{2}=\frac{4}{\theta^{2} \mu^{2}} e^{-8 \pi / \tilde{\lambda}(\mu)}
$$

Modes of $\phi(x)$ with momenta less than this value are tachyonic, a result of the instability caused by not using a proper solution of the gap equation.

\subsection{A double scaling limit}

Quite interesting things happen in the double scaling limit when $\Lambda \rightarrow \infty$ and $\theta \rightarrow 0$ so that $\Lambda \theta=C / M$ with an arbitrary constant $C$. The physical meaning of this limit is that one "regularizes" the ordinary Gross-Neveu model by a noncommutative one at the cutoff scale. This theory is non-commutative only at distance scales of order of and smaller than the ultraviolet cutoff. But in field theory, the UV/IR mixing of large and small momentum scales means that it still has an effect. In this particular limit we can obtain an exact expression:

$$
\lambda^{\mathrm{eff}}(q)=\frac{4 \pi}{\frac{1}{2} \ln \left(1+C^{2} q^{2} / M^{2}\right)+\frac{\sqrt{1+\frac{q^{2}}{4 M^{2}}}}{\frac{q}{2 M}} \ln \left(\sqrt{1+\frac{q^{2}}{4 M^{2}}}+\frac{q}{2 M}\right)} .
$$

The second term in the denominator has a square root cut starting from $q=2 M i$ in the complex $q$ plane, which corresponds to a pair production of fermions. This is the same as what occurs in the commutative Gross-Neveu model. What is new and interesting is the first term. It has a logarithmic cut starting from $q=i M / C$. This cut is absent in the commutative model. We speculate that it corresponds to creation of pairs of some non-local solitons present in the non-commutative theory, which survive this double scaling limit. However, as yet we have not been 
able to find an explicit form for the objects which are created, if indeed they are solitons.

We see that the limits $\Lambda \rightarrow \infty$ and $\theta \rightarrow 0$ do not commute and even in the case when non-commutativity is relevant at the cutoff scale it still modifies the behavior of the theory at any energy scale.

\section{Results in three dimensions}

In the three dimensional Gross-Neveu model, the coupling constant $\lambda$ has the dimension of length. This model is therefore not renormalizable in a perturbation theory in the coupling constant. However, in the large $N$ expansion of the commutative model, the only counterterms needed to cancel the divergences that arise are of the same form as the operators which are already in the action. Thus, by the usual definition, the commutative theory is a renormalizable model in the $1 / N$ expansion. The reason for its renormalizability is the existence of a second order phase transition which occurs at a sufficiently large value of the coupling constant. It is near that value of the coupling that the theory is renormalizable. At the second order phase transition, the four-fermion coupling has a large anomalous dimension, so it is effectively dimensionless 22].

In three dimensions, the gap equation (21) with a suitable cutoff is

$$
0=M\left[\frac{1}{\lambda}-\frac{1}{16 \pi} \Lambda \exp \left(-\frac{2 M}{\Lambda}\right)\right]
$$

Unlike in two dimensions, we see that in three dimensions, which of the two possible solutions of (37) is stable, depends on the size of the coupling constant. If

$$
\lambda<\frac{16 \pi}{\Lambda}
$$

the solution is

$$
M=0
$$

In this phase, the fermions are massless and the chiral symmetry is unbroken.

If, on the other hand,

$$
\lambda>\frac{16 \pi}{\Lambda}
$$

then $M$ solves the equation

$$
\frac{1}{\lambda}=\frac{1}{16 \pi} \Lambda \exp \left(-\frac{2 M}{\Lambda}\right) .
$$

In this phase, the fermions have mass and the chiral symmetry is spontaneously broken.

\subsection{Commutative model}

Let us first examine the commutative model. There are two phases which are separated by a second order phase transition. 


\subsubsection{Symmetric, massless phase}

First, consider the symmetric phase, where $\lambda<16 \pi / \Lambda$. In this case, the correlation function $\tau_{\mathrm{c}}(q)$ which determines the effective coupling is readily computed,

$$
\tau_{\mathrm{c}}(q)=-\frac{\Lambda}{16 \pi}+\frac{|q|}{16}
$$

and the effective coupling constant is

$$
\lambda^{e f f}(q)=\frac{1}{\frac{1}{\lambda}-\frac{\Lambda}{16 \pi}+\frac{|q|}{16}}
$$

We tune $\lambda$ to be sufficiently close to the critical value, and of course less than the critical value,

$$
\frac{1}{\lambda}=\frac{\Lambda}{16 \pi}+\frac{\mu^{2}}{16} \quad, \quad \mu \geq 0
$$

This is equivalent to adding a counterterm to the action. The result is

$$
\lambda^{e f f}(q)=\frac{16}{\mu^{2}+|q|}
$$

At the critical point, when $\mu^{2}=0$ this is a non-trivial conformal field theory whose scaling properties can be computed order by order in the $1 / N$ expansion 22, 23, 24. At the critical point, the fermion mass operator $\bar{\psi} \psi$ has conformal dimension 1, rather than its tree level value of 2 .

Note that, in the large $N$ expansion, the bare coupling constant behaves as it would in an asymptotically free theory. If we hold the finite mass scale $\mu$ fixed as we put the ultraviolet cutoff $\Lambda$ to infinity the bare coupling $\lambda$ runs to zero. In this sense, in the large $N$ expansion, the three dimensional Gross-Neveu model behaves as if it were an asymptotically free theory.

\subsubsection{Massive phase with broken symmetry}

Now, we consider the regime where $\lambda>16 \pi / \Lambda$ where the gap equation for $M$ has a nonzero solution. The correlator $\tau(p)$ for this case can be computed. It is

$$
\tau_{\mathrm{c}}(q)=-\frac{1}{16 \pi} \Lambda e^{-\frac{2 M}{\Lambda}}+\frac{1}{4 \pi} \frac{4 M^{2}+q^{2}}{|q|} \arctan \frac{|q|}{2 M}
$$

We combine this with the gap equation to find the effective coupling constant

$$
\lambda_{\mathrm{c}}^{\mathrm{eff}}(q)=\frac{4 \pi}{\frac{4 M^{2}+q^{2}}{|q|} \arctan \frac{|q|}{2 M}}
$$

This coupling constant goes to zero like $\lambda_{\mathrm{c}}^{\mathrm{eff}} \sim 16 /|q|$ for large $\mathrm{q}$ and has a perfectly finite constant limit for small $\mathrm{q}, \lambda_{\mathrm{c}}^{\mathrm{eff}} \approx 4 \pi / M$. We note that both the cutoff and the bare coupling constant have disappeared from this equation. It depends only on the fermion mass $M$. This is the analog of dimensional transmutation in this three dimensional model. 


\subsection{Non-commutative theory}

In the non-commutative theory, there are two contributions to the correlator $\tau(q)$, one from a planar diagram, which gives the same result as in the commutative theory with an over all factor of $1 / 2$,

$$
\tau_{n c}^{\mathrm{pl}}(q)=-\frac{1}{32 \pi} \Lambda e^{-\frac{2 M}{\Lambda}}+\frac{1}{8 \pi} \frac{4 M^{2}+q^{2}}{|q|} \arcsin \frac{|q|}{\sqrt{4 M^{2}+q^{2}}}
$$

The other is from a non-planar diagram and depends explicitly on the noncommutativity parameter,

$$
\begin{array}{r}
\tau_{\mathrm{nc}}^{\mathrm{nonpl}}(q)=-\frac{1}{32 \pi}\left(\frac{|\theta q|^{2}}{4}+\frac{1}{\Lambda^{2}}\right)^{-\frac{1}{2}} \exp \left[-2 M\left(\frac{|\theta q|^{2}}{4}+\frac{1}{\Lambda^{2}}\right)^{\frac{1}{2}}\right]+ \\
+\frac{1}{16 \pi}\left(4 M^{2}+q^{2}\right) \int_{0}^{1} \frac{d \alpha}{\sqrt{M^{2}+\alpha(1-\alpha) q^{2}}} \exp \left(-|\theta q| \sqrt{M^{2}+\alpha(1-\alpha) q^{2}}\right)
\end{array}
$$

If we assume that the gap equation is satisfied, this theory also has two different phases, which we can study separately.

\subsubsection{Symmetric, massless phase}

As in the commutative theory, if $\lambda<16 \pi / \Lambda$, the fermion mass is zero, $M=0$. In this case we can use the massless limits of the equations (43) and (44) which are

$$
\tau_{\mathrm{nc}}^{\mathrm{nonpl}}(q)=-\frac{1}{32 \pi} \Lambda+\frac{|q|}{16}
$$

and

$$
\tau_{\mathrm{nc}}^{\mathrm{nonpl}}(q)=\frac{-1}{32 \pi}\left(\frac{|\theta q|^{2}}{4}+\frac{1}{\Lambda^{2}}\right)^{-\frac{1}{2}}+\frac{|q|}{16 \pi} \int_{0}^{1} \frac{d \alpha}{\sqrt{\alpha(1-\alpha)}} \exp (-|\theta q||q| \sqrt{\alpha(1-\alpha)})
$$

Then, the effective interaction is given by the equation

$$
\begin{gathered}
\frac{1}{\lambda^{\mathrm{eff}}(q)}=\frac{1}{\lambda}-\frac{\Lambda}{32 \pi}+\frac{|q|}{16}-\frac{1}{32 \pi}\left(\frac{|\theta q|^{2}}{4}+\frac{1}{\Lambda^{2}}\right)^{-\frac{1}{2}}+ \\
\quad+\frac{|q|}{16 \pi} \int_{0}^{1} \frac{d \alpha}{\sqrt{\alpha(1-\alpha)}} \exp (-|\theta q||q| \sqrt{\alpha(1-\alpha)}) .
\end{gathered}
$$

where we have kept the cutoff dependence in the third term on the right-handside to regulate the behavior of this term at small $q$. Now the question is, can we choose a cut-off dependent $1 / \lambda$ in such a way that the large $\Lambda$ limit can be taken?

The answer is no. The reason is because the right-hand-side of (47) has a minimum when $q=0$, so the smallest that we can make it is to choose $1 / \lambda$ to cancel its value at $q=0$. This choice is

$$
\frac{1}{\lambda}=\frac{\Lambda}{16 \pi}+\mu^{2}
$$


Here, $\mu^{2}$ has to be positive if we are to be in the massless phase, then $\mu^{2} \rightarrow 0$ the coupling constant is at the critical point.

$$
\begin{gathered}
\frac{1}{\lambda_{\mathrm{c}}^{\mathrm{eff}}(q)}=\mu^{2}+\frac{|q|}{16}+\frac{1}{32 \pi}\left(\Lambda-\frac{1}{\sqrt{\frac{|\theta q|^{2}}{4}+\frac{1}{\Lambda^{2}}}}\right)^{-\frac{1}{2}}+ \\
\quad+\frac{|q|}{16 \pi} \int_{0}^{1} \frac{d \alpha}{\sqrt{\alpha(1-\alpha)}} \exp (-|\theta q||q| \sqrt{\alpha(1-\alpha)}) .
\end{gathered}
$$

This effective coupling is positive for all values of $q$. This is desirable, as this means that it does not have a Landau pole singularity.

However, the cutoff dependence is still there. For any non-zero value of $q$ it behaves like

$$
\lambda^{\mathrm{eff}}(q) \approx \frac{32 \pi}{\Lambda}
$$

which goes to zero as $\Lambda \rightarrow \infty$. The theory has a trivial effective coupling constant.

There is no freedom to further adjust $\mu^{2}$ so that the cutoff dependence cancels since, to be in the massless phase, it must be positive. If we did, naively try to choose $1 / \lambda$ so as to cancel all of the cutoff dependence when the momentum is finite. $\frac{1}{\lambda}=\frac{\Lambda}{32 \pi}+\mu^{2}$ we would discover that the massless ground state is unstable, that modes with momentum in the range $|\theta q| \approx 1 / 16 \pi \mu^{2}$ are tachyonic.

\subsubsection{Double Scaling limit}

As in the two dimensional case, there is a limit where the cutoff can be decoupled. Assume that $\theta^{0 i}=0$ and $\theta^{i j}=\epsilon^{i j} \theta$ where $\epsilon^{i j}$ is the fully antisymmetric tensor with $\epsilon^{01}=1$. We consider the limit where we take $\Lambda \rightarrow \infty$ and then we keep $\Lambda^{3} \theta^{2}$ a fixed finite number with momentum dimension -1 . In that case,

$$
\frac{1}{\lambda_{\text {eff }}(q)}=\frac{1}{\tilde{\lambda}}+\frac{q}{8}+\frac{\Lambda^{3} \theta^{2} \vec{q}^{2}}{256 \pi}
$$

This means that the force between the fermions is mediated by exchange of a particle with non-relativistic dispersion relation

$$
\omega(\vec{q})=|\vec{q}| \sqrt{\vec{q}^{2}+\Lambda^{3} \theta^{2} / 32 \pi}
$$

It is long-ranged, but no longer scale invariant. In this case, even though the non-commutativity occurs only at distance scales that have been excised by the ultraviolet cutoff, there is still some remnant of it caused by the ultraviolet divergences of the field theory.

This limit has rather severe implications when we consider the space-space uncertainty relation $\Delta x \Delta y=\theta$ which we re-write as $\Delta x \Delta y=\left(\Lambda^{3 / 2} \theta\right) / \Lambda^{3 / 2}$. If $\Delta x$ is finite, then, there is a coordinate-momentum uncertainty relation $\Delta x \Delta p_{x} \sim$ $1 \Delta y \sim\left(\Lambda^{3 / 2} \theta\right) \Delta p_{x} / \Lambda^{3 / 2}$ is smaller than the short distance cutoff which is of size $1 / \Lambda$. The uncertainty relation should therefore be invisible in the cutoff theory. Note that in the two dimensional theory it would have been of order the small distance cutoff and marginally observable. 


\subsubsection{Gapped phase with broken symmetry}

The massive phase of this theory suffers much the same fate as the two-dimensional model. The cutoff dependence of the bare coupling constant is dictated by the gap equation. We must solve the gap equation to get a stable solution. Then some cutoff dependence remains in the effective coupling constant, From (43), (44) one immediately sees that for momenta above $|\theta q| \sim 1 / \Lambda$ the dependence on momentum in $\lambda^{\text {eff }}(q)$ is a small correction in comparison with the term $\sim \Lambda$. Hence, we have

$$
\lambda_{\mathrm{c}}^{\mathrm{eff}}(q) \approx \frac{1}{\frac{1}{\lambda}-\frac{1}{32 \pi} \Lambda e^{-\frac{2 M}{\Lambda}}}=\frac{32 \pi}{\Lambda} e^{\frac{2 M}{\Lambda}},
$$

where $\lambda$ is eliminated using (28). Hence, in this phase we get a situation similar to the 2-dimensional Gross-Neveu model, except that the cutoff dependence is more severe - it effective coupling goes to zero linearly, rather than logarithmically as the cutoff is put to infinity.

\section{Conclusions}

We have examined the issue of renormalizability of the large $N$ expansion of the non-commutative $O(N)$ Gross-Neveu models in 2 and 3 space-time dimensions. In both cases, we find that the ultraviolet cutoff does not decouple from the theory. If we take it to infinity, the four-fermion interaction becomes trivial.

There is a question as to whether this is a generic feature of non-commutative field theories with translationary invariant vacua. Of course, UV/IR mixing is generic. Consider the following general physical arguments. An excitation with large momentum $p_{x}$ in a non-commutative theory has uncertainty in its position along the momentum $\Delta x \sim 1 / p_{x}$ which is very small. Hence, taking into account that $x$ and $y$ coordinates do not commute, we see that the uncertainty in $y$ is very big. This mixes IR and UV limits in the sense that IR effects modify UV limit and vice-versa [26]. This mixing is seen in our example as well as others as the appearance of small momentum singularities in primitively divergent correlation functions which are therefore also generic.

Generally, and especially in systems where the effective coupling is strong in the infrared, the strong coupling dynamics generates a mass gap. In a commutative theory this generation of a mass gap is what cures the problems, such as the infrared Landau pole, which occurs in theories where the coupling constant has no infrared stable fixed point. In this Paper we have seen an explicit example of a non-commutative theory where the same mechanism - the generation of a mass gap - does not cure the problems associated with strong coupling.

Non-commutative gauge theories are often given as examples of field theories which come from string theory in a strong field and zero slope limit. For example, in 28] it was claimed that one can obtain asymptotically free non-commutative

SUSY Yang-Mills theories by a self-consistent truncation of the massive string 
modes. In this paper they extended tree level considerations of [12] to the case of loop corrections. It would be interesting to check whether a consistent noncommutative theory really results. This is a very non-perturbative question 27] and is complicated by the lack of any local gauge invariant observables in noncommutative Yang-Mills theory [29] (see also [30]).

It is worth mentioning that we are aware of non-commutative theories which are renormalizable because $\theta$ does not enter into divergent graphs, i.e. noncommutativity does not modify UV properties of the theories. The list of such theories includes Gross-Neveu model with Dirac fermions and non-commutative $N=4$ SYM theory living on the D3-brane in type IIB string theory with constant $B$-field background. In these cases, because the ultraviolet divergences are unaffected by non-commutativity, the infrared singularities that would appear with them are also absent.

An interesting aspect of our considerations for asymptotically free non-commutative theories with $\theta$ parameter entering into divergent diagrams is the assumption that the vacuum is translationally invariant. It indeed seems to be a reasonable assumption since the effective action has a deep minimum for translationally invariant states.

After this paper was written, it was pointed out to us that there is some overlap between our work and that contained in [31]. In fact, they confirm our analysis of the Gross-Neveu model and they also show that the 2-dimensional asymptotically free sigma models also have non-renormalizable large $\mathrm{N}$ expansions.

This work is supported in part by NSERC of Canada. E.T.A. was supported by a NATO Science Fellowship and grants INTAS-97-01-03 and RFBR 98-02-16575 and G.S and E.T.A were supported in part by NATO Collaborative Linkage Grant SA(PST.CLG.977361)5941. We thank Kostya Zarembo and Yurii Makkenko for valuable discussions.

\section{References}

[1] E. T. Akhmedov, P. DeBoer and G. W. Semenoff, "Running couplings and triviality of field theories on non-commutative spaces," hep-th/0010003.

[2] D. J. Gross and A. Neveu, "Dynamical Symmetry Breaking In Asymptotically Free Field Theories," Phys. Rev. D10, 3235 (1974).

[3] G. Parisi, "Some Considerations On Nonrenormalizable Interactions," in C74-06-24.1.2 INFN-ROME-573 Presented at Colloquium on Lagrangian Field Theory, Marseille.

[4] D. J. Gross, "Applications Of The Renormalization Group To High-Energy Physics," In *Les Houches 1975, Proceedings, Methods In Field Theory*, Amsterdam 1976, 141-250.

[5] B. Rosenstein, B. J. Warr and S. H. Park, "The Four Fermi Theory Is Renormalizable In (2+1)-Dimensions," Phys. Rev. Lett. 62, 1433 (1989). 
[6] G. W. Semenoff and L. C. Wijewardhana, "Dynamical violation of parity and chiral symmetry in 3-D four Fermi theory," Phys. Rev. D45, 1342 (1992).

[7] G. W. Semenoff and L. C. Wijewardhana, "Dynamical Mass Generation In 3-D Four Fermion Theory," Phys. Rev. Lett. 63, 2633 (1989).

[8] J. Gomis and T. Mehen, "Space-time noncommutative field theories and unitarity," hep-th/0005129.

[9] P. Ho and Y. Wu, "Noncommutative geometry and D-branes," Phys. Lett. B398, 52 (1997) hep-th/9611233.

[10] A. Connes, M. R. Douglas and A. Schwarz, "Noncommutative geometry and matrix theory: Compactification on tori," JHEP 9802, 003 (1998) hepth/9711162.

[11] C. Chu and P. Ho, "Constrained quantization of open string in background B field and noncommutative D-brane," Nucl. Phys. B568, 447 (2000) hepth/9906192.

[12] N. Seiberg and E. Witten, "String theory and noncommutative geometry," JHEP 9909, 032 (1999) hep-th/9908142.

[13] T. Lee, "Canonical quantization of open string and noncommutative geometry," Phys. Rev. D62, 024022 (2000) hep-th/9911140.

[14] M. Laidlaw, "Noncommutative geometry from string theory: Annulus corrections," hep-th/0009068.

[15] S. Minwalla, M. Van Raamsdonk and N. Seiberg, "Noncommutative perturbative dynamics," hep-th/9912072.

[16] M. Van Raamsdonk and N. Seiberg, "Comments on noncommutative perturbative dynamics," JHEP 0003, 035 (2000) hep-th/0002186].

[17] T. Filk, "Divergencies in a field theory on quantum space," Phys. Lett. B376, 53 (1996).

[18] S. S. Gubser and S. L. Sondhi, "Phase structure of non-commutative scalar field theories," hep-th/0006119.

[19] I. Chepelev and R. Roiban, "Renormalization of quantum field theories on noncommutative $\mathrm{R}^{* *}$ d. I: Scalars," JHEP 0005, 037 (2000) hepth/9911098].

[20] I. Chepelev and R. Roiban, "Convergence theorem for non-commutative Feynman graphs and renormalization," hep-th/0008090.

[21] A. Micu and M.Sheikh-Jabbari, "Non-commutative $\Phi^{4}$ theory at two loops", hep-th/0008057.

[22] W. Chen, Y. Makeenko and G. W. Semenoff, "Four fermion theory and the conformal bootstrap," Annals Phys. 228, 341 (1993) hep-th/9301069].

[23] A. Petkou, "Conserved currents, consistency relations, and operator product expansions in the conformally invariant $\mathrm{O}(\mathrm{N})$ vector model," Annals Phys. 249, 180 (1996) [hep-th/9410093.

[24] A. C. Petkou and M. B. Silva Neto, "On the free energy of three-dimensional CFTs and polylogarithms," Phys. Lett. B456, 147 (1999) hep-th/9812166].

[25] J. Ambjorn, Y. M. Makeenko, J. Nishimura and R. J. Szabo, "Nonperturbative dynamics of noncommutative gauge theory," Phys. Lett. B480, 399 (2000) hep-th/0002158. 
[26] A. Matusis, L. Susskind and N. Toumbas, "The IR/UV connection in the non-commutative gauge theories," JHEP0012, 002 (2000) hep-th/0002075.

[27] V. V. Khoze and G. Travaglini, "Wilsonian effective actions and the IR/UV mixing in noncommutative gauge theories," JHEP0101, 026 (2001) hepth/0011218.

[28] J. Gomis, M. Kleban, T. Mehen, M. Rangamani and S. Shenker, "Noncommutative gauge dynamics from the string worldsheet," JHEP0008, 011 (2000) hep-th/0003215.

[29] D. J. Gross, A. Hashimoto and N. Itzhaki, "Observables of non-commutative gauge theories," hep-th/0008075.

[30] D. Berenstein and R. G. Leigh, "Observations on non-commutative field theories in coordinate space," hep-th/0102158.

[31] H. O. Girotti, M. Gomes, V. O. Rivelles and A. J. da Silva, "The noncommutative supersymmetric nonlinear sigma model," hep-th/0102101. 